Original Article

\title{
IMPACT OF LIFESTYLE ON THE OCCURRENCE OF ANXIETY AMONGST THE RESIDENTS OF UNITED ARAB EMIRATES
}

\author{
MUAED JAMAL ALOMAR, AMAL MOHAMED ABDI, SUMAIYA ZAMAN, HANADI MOHAMED ABDI, M. A. \\ FRANCHESKA QUICHO
}

College of Pharmacy and Health Sciences-Ajman University

Email: muayyad74@yahoo.com

Received: 10 Jul 2017 Revised and Accepted: 04 Jan 2018

\begin{abstract}
Objective: To investigate the effect of lifestyle on the occurrence of anxiety. Also, to study the determinants of the severity of anxiety.

Methods: A cross-sectional survey was carried out on 100 randomly selected participants.

Results: Many factors were taken into consideration in this study including gender, age, occupational status, marital status, eating habits, physical activity, psychological stress, sleep patterns, medications and smoking habits. Subanalysis of some of the results showed a statistical significance of different aspects of lifestyle affecting levels of anxiety. According to the results found, the lifestyle factors that have significance in the severity of anxiety are psychological stress, exercising, and regularity of exercising (Pearson's Chi-Square test: $\mathrm{P}<0.001$, Pearson's R: $\mathrm{P}=0.027$, Spearman Correlation: $\mathrm{P}=0.045$ respectively). About $50 \%$ of participants that stated they have some kind of psychological stress fell within the range of severe to very severe anxiety. The majority of those who exercise (39\%) fall in the normal range of anxiety, and the majority of those who do not exercise (34\%) are in the very severe anxiety range. Among the 14 participants that stated they exercise every day, $46 \%$ of them were in the normal range of anxiety while none of them was in the very-severe range. Also, those who exercise a few times a month are ranged more in the severe anxiety level than those who exercise every day.
\end{abstract}

Conclusion: Certain lifestyle factors affect the levels of anxiety in various ways. This research clearly identified that a significant percentage of people who have any sort of psychological stress and do not exercise regularly are at a higher risk of developing or deteriorating anxiety.

Keywords: Anxiety, Lifestyle, DASS42

(c) 2018 The Authors. Published by Innovare Academic Sciences Pvt Ltd. This is an open-access article under the CC BY license (http://creativecommons.org/licenses/by/4.0/) DOI: http://dx.doi.org/10.22159/ijpps.2018v10i2.21241

\section{INTRODUCTION}

Anxiety may be helpful sometimes as it is considered a natural reaction of the body to stress hence making an individual highly alert and attentive. It is the fear or nervousness about what might happen; feeling this way occasionally is normal, but if anxiety starts to interfere with daily life, that might be an indication of a disorder [1].

The major causes of the symptoms of anxiety disorder in the central nervous system are serotonin, norepinephrine, dopamine, and gamma-aminobutyric acid (GABA)[2]. Also, other peptides and neurotransmitters such as corticotrophin-releasing factor can cause anxiety. When the corticotropin-releasing factor is administered centrally, it causes behavioural and psychological alternations. According to clinical studies, overproduction of CRF have proved to play a role in anxiety disorder [3].

Depression and anxiety are considered the most two common health mental problems. Although more attention is given to anxiety, it is also considered a reason for depression in research and clinical studies for screening, diagnosis, and treatment of affected individuals, unfortunately giving rise to the propagation of anxiety and its effect on patient life and work productivity [4].

Real factors include age, gender, economic status, culture, conflict and the urban city considered the greatest proportion of variability in anxiety therapy [5]. The current global extensiveness of anxiety disorder adjusted for methodological differences was 7.3\% (4.8$10.9 \%$ ) and in African cultures, it ranged from 5.3\% (3.5\%-8.1\%) to $10.4 \%$ (7.0-15.5\%) in the Euro/Anglo cultures [5].

Millions of people around the world experience anxiety problems. In the USA, anxiety disorders affect 15.7 million people each year. Also, 30 million in US at some point during their lifetime experience anxiety [6]. The total cost of treatment courses in United States is about 42 billion dollars per year. The most common type of anxiety disorder is a social anxiety disorder or which is known as social phobia affecting 5 million people. This is followed by generalized anxiety (GAD) and posttraumatic stress disorder (PSTD). Approximately 1 in 30-50 people suffer from obsessive-compulsive disorder (OCD) and 1 in 10 reported to have some specific phobia [7]. Epidemiological Catchment Area (ECA) study estimated lifetime prevalence rates for panic disorder (2.3-2.7\%), (4.1-6.6\%) for generalized anxiety disorder and (2.6-13.3\%) for social phobia [7]. In Germany, anxiety disorder was found to affect $18.6 \%$ of adolescents of which phobia was the most common. Other types of anxiety-like post-traumatic stress disorder and obsessive-compulsive disorder rates were below $2 \%$. Generalized anxiety disorder and panic disorder was the least common with rates below $1 \%$. They also found that anxiety was higher in girls than in the boys and there was an increase in the rate with age [8]. Anxiety disorders are considered one of the most common psychiatric diseases in children, 1 in 3 are actually suffering from anxiety at some point through adolescence or childhood [9]. Significant impairment to quality of life was found among individuals with subthreshold styles of anxiety disorders [10]. The epidemiological evidence stated that people with psychiatric disorders, especially with social phobia, are more likely to withdraw from school prematurely [11]. 201 patients who met the DSM-IV criteria, completed a questionnaire and the results proved that almost $49 \%$ of students drop out of high school. $24 \%$ of them reported that anxiety was the main cause behind their decision [12]. Generalized anxiety disorder is a long-lasting excessive and irrational worry over things such as the personal safety, health state, and finance issue. Trembling, various body aches and pain are some physical symptoms associated to generalized anxiety disorder [13]. Panic disorder is an attack that comes on for no apparent reason. Personal experiences are one of the major causes of anxiety disorder along with environmental factors or genetic factors. Anxiety disorder can be inherited and sometimes runs in the family. Usually, children whose parents have anxiety disorder have a greater chance of developing 
anxiety disorders. Sometimes, traumatic events, such as death, may cause anxiety to develop in certain people. However, not every individual who experiences these events suffers from anxiety and not everyone that suffers from anxiety has faced traumatic events in their life. Genetic factors do not seem to cause generalized anxiety disorder. While other anxiety disorders, mostly agoraphobia with panic attacks and panic disorder could happen due to genetic factors [14].

The aim of this research is to evaluate the effect of lifestyle on the occurrence of anxiety amongst the population of various emirates across UAE.

\section{MATERIALS AND METHODS}

A prospective cross-sectional survey amongst the population of UAE was carried out using simple random sampling technique selecting 100 participants within specific inclusion criteria. The study was conducted between February 2017 and April 2017. Criteria of the questionnaire included: demographic data (gender, age, occupation, and marital status), lifestyle data (physical activity, eating habits, sleep pattern, psychological stress, medications and smoking habits) and questions to evaluate the level of anxiety using the anxietybased questions on DASS42. The data was collected via both distributions of the questionnaire and online based questionnaire. After the collection of data, SPSS was used to analyze the results. Pearson's chi-square test was used to determine the significance of interactions with P-value $<0.05$.

\section{RESULTS}

\section{Demographic data}

Demographics of the participants are represented in the table 1 below, where (33.0\%) of them were male and the rest (67.0\%) were female. As for their age, $(28.0 \%)$ of them were aged 20 and under, the majority $(56.0 \%)$ were aged $21-25,(3.0 \%)$ were around $26-30$ and finally, (13.0\%) of them were 31 and over. As for the occupation, $(82.0 \%)$ of them were students, $(17.0 \%)$ Employed and $(1.0 \%)$ were unemployed. $(82.0 \%)$ of the participants were single and the $(15.0 \%)$ were married while $3 \%$ were either engaged or divorced.

Table 1: Demographic data

\begin{tabular}{ll}
\hline Gender & Percentage \\
\hline Male & $33 \%$ \\
Female & $67 \%$ \\
Age: & Percentage \\
20 and under & $28 \%$ \\
$21-25$ & $56 \%$ \\
$26-30$ & $3 \%$ \\
31 and over & $13 \%$ \\
Occupation & Percentage \\
Student & $81 \%$ \\
Employed & $15 \%$ \\
Unemployed & $4 \%$ \\
Marital status & Percentage \\
Single & $82 \%$ \\
Married & $15 \%$ \\
Other & $3 \%$ \\
\hline
\end{tabular}

\section{Eating habits}

Table 2 shows the eating habits of the participants, The majority $(19.0 \%)$ seemed to eat carbohydrates, whereas only $(6.0 \%)$ ate only proteins and the rest were fats only(3.0\%), fruits and vegetables only(5.0\%), Cand P (9.0\%), CandF (6.0\%), CandFV (3.0\%), PandF
(1.0\%), PandFV (4.0\%), CandPandF (10.0\%), CandPandFV (11.0\%), PandFandFV (2.0\%), CandPandFandFV $(18.0 \%)$, CandFandFV $(3.0 \%)$. And it was found that huge majority of $(96.0 \%)$ eats out with most $(35.0 \%)$ eating out few times a month, closely followed by $(31.0 \%)$ eating out $1-2$ times a week, $(23.0 \%)$ eating out more than twice a week and (8.0\%) eats out almost every day.

Table 2: Eating habits

\begin{tabular}{ll}
\hline Types of food & Percentage \\
\hline Carbohydrates & $19 \%$ \\
Proteins & $6 \%$ \\
Fats & $3 \%$ \\
Fruits and vegetables & $5 \%$ \\
C and P & $9 \%$ \\
C and F & $6 \%$ \\
C and FV & $3 \%$ \\
P and F & $1 \%$ \\
P and FV & $4 \%$ \\
C and P and F & $10 \%$ \\
C and P and FV & $11 \%$ \\
P and F and FV & $2 \%$ \\
C and P and F and FV & $18 \%$ \\
C and F and FV & $3 \%$ \\
Eating out & \\
Yes & $96 \%$ \\
No & $4 \%$ \\
How often & \\
FEW times a month & $35 \%$ \\
$1-2$ times a week & $31 \%$ \\
More than twice a week & $23 \%$ \\
Almost everyday & $8 \%$ \\
\hline
\end{tabular}

C: Carbohydrates, P: Protein, F: Fat, FV: Fruits and Vegetables 


\section{Physical activity}

Table 3 demonstrates the physical activity of the participants. The vast majority of the participants highlighted that they do exercise $(71.0 \%)$ and about $(29.0 \%)$ said that they do not. $23 \%$ do so only a few times a month. (19.0\%) of the participants claimed to exercise 1-2 times a week, $(15.0 \%)$ of the participants said they exercise more than twice a week and $(14.0 \%)$ stated that they exercised every day. When inquired about the type of exercise $(42.0 \%)$ said that they simply walk/run/jog, (18.0\%) prefer swimming and rest of the $(8.0 \%)$ undertook various other exercises.
Sleeping habit; $46 \%$ admitted that they do not get enough sleep while $54 \%$ claimed that they do.

Stress problems; $77 \%$ percent have psychological stress whereas $23 \%$ did not suffer from any psychological stress.

Medications; 83\% percent of the participants reported that they do not take medications in comparison to only $17 \%$ of those who do.

Smoking habit; The vast majority of the participants highlighted that they do not smoke (86\%) while $14 \%$ of them were smokers.

Table 3: Physical activity

\begin{tabular}{ll}
\hline Do you exercise & Percentage \\
\hline Yes & $71 \%$ \\
No & $29 \%$ \\
How often & \\
Few times a month & $23 \%$ \\
$1-2$ times a week & $19 \%$ \\
More than twice a week & $15 \%$ \\
Everyday & $14 \%$ \\
Type of exercise & $42 \%$ \\
Walking/Running/Jogging & $18 \%$ \\
Gym & \\
Swimming & \\
Other (specify) & \\
\hline
\end{tabular}

\section{Comparison between lifestyle factors and its effect on anxiety level}

Comparison between lifestyle factors and anxiety level was assessed by asking the participants all the anxiety-based questions on DASS42. Analysis run to assess the research was done using the Pearson's Chi-square test.
There are very few lifestyle factors that can affect the level of anxiety. Among these factors, psychological stress played a huge role in the extent of anxiety with a chi-square significant value of $\mathrm{P}<0.001$. However, no significance was found between other lifestyle factors and anxiety. The other P-values related to lifestyle and anxiety are demonstrated in table 4.

Table 4: Demographics and lifestyle factors

\begin{tabular}{|c|c|c|}
\hline Variable & Statistical test & P-value \\
\hline Gender & Pearson Chi-Square & $\mathrm{P}=0.746$ \\
\hline Age & Pearson Chi-Square & $P=0.705$ \\
\hline Occupation & Pearson Chi-Square & $P=0.358$ \\
\hline Marital status & Pearson Chi-Square & $\mathrm{P}=0.684$ \\
\hline Types of food & Pearson Chi-Square & $\mathrm{P}=0.462$ \\
\hline Eating out & Pearson Chi-Square & $P=0.678$ \\
\hline Frequency of eating out & Pearson Chi-Square & $\mathrm{P}=0.271$ \\
\hline \multirow[t]{3}{*}{ Exercising } & Linear-by-Linear Association & $\cdot P=0.043$ \\
\hline & Pearson's R & $\cdot P=0.042$ \\
\hline & Spearman Correlation & $\cdot P=0.049$ \\
\hline \multirow[t]{3}{*}{ Frequency of exercising } & Likelihood Ratio & $\cdot P=0.059$ \\
\hline & Linear-by-Linear Association & $\cdot P=0.026$ \\
\hline & - Spearman Correlation & $\cdot P=0.045$ \\
\hline Enough sleep & Pearson Chi-Square & $P=0.609$ \\
\hline Psychological stress & Pearson Chi-Square & $P=0.001$ \\
\hline Medication & Pearson Chi-Square & $\mathrm{P}=0.903$ \\
\hline Smoking & Pearson Chi-Square & $\mathrm{P}=0.227$ \\
\hline
\end{tabular}

Table 4 shows the impact of certain factors on the level of anxiety

\section{DISCUSSION}

As a random number of participants took part in the study it was found that different lifestyles may or may not affect an individual's anxiety level. All participants were approached regardless of their age, gender, marital status and occupational status. There was significant difference found between some lifestyle factors and anxiety while some factors did not portray any significant difference.

The results of this study show that psychological stress plays the most major role in an individual's level of anxiety with a $\mathrm{P}$ value of $\mathrm{P}=0.001$. Almost $50 \%$ of those who stated that they have some kind of psychological stress fell within the range of severe to very severe anxiety. These results are comparable with another study done on 38 medical students based on stress-induced anxiety that showed students with high stress had a significantly higher anxiety level [15].

On the other hand, it was found that gender did not play a huge role in the occurrence of anxiety but females are more prone to developing anxiety with $25 \%$ of the female participant having very severe anxiety whereas only $13 \%$ of the male participants suffered from the same. This study is in sync with another study that reported the constancy of anxiety disorders did not show any difference across genders [16]. Furthermore, out of 71 participants who reported that they exercise $49 \%$ of them fell in the normal to mild range of anxiety and out of the 29 respondents who reported that they do not exercise, $55 \%$ of them 
where in the severe to very-severe range of anxiety (exercise vs. level of anxiety, Pearson's R Sig. $\mathrm{P}=0.042$ ). In addition, this study also investigated the importance of the regularity of exercising. Among the 14 participants that stated they exercise every day, $46 \%$ of them were in the normal range of anxiety while none of them was in the verysevere range. Along with more results indicating that those who exercise a few times a month are ranged more in the severe anxiety level than those who exercise every day indicating that not only exercising but also the frequency of exercising may help relieve or prevent anxiety (regularity of exercise vs. level of anxiety, Pearson's R Sig. $\mathrm{P}=0.025$ ). Preceding studies have shown that at least fifteen minutes of physical activity or 20 min physical activity in an aerobic environment may reduce daily anxiety [17]. Moreover, the results shows that about $41 \%$ of the participant who claimed to get enough sleep fell in the normal range while $28 \%$ who experienced severe anxiety claimed they did not get enough sleep. This study is supported by another study on 88 physically and psychologically healthy adults concluded that increased anxiety is caused by lack of sleep. Sleep is consistently interrupted in people who are suffering from anxiety disorders [17].

In fact, according to age-related anxiety, 35\% of the $20 \mathrm{y}$ and under participants suffered from very-severe anxiety, in comparison to $23 \%$ of the $31 \mathrm{y}$ and over. This study is consistent with another study done on a large group of people with ages between 30 s to 65 and over concluded that anxiety disorders are less pervasive in older individuals than younger, showing also an age-related decline in the prevalence of anxiety disorders [18].

Moreover, this study covers the impact of marital status on the level of anxiety. From the results obtained, a person's marital status does not really affect their anxiety as $40 \%$ of those who are both single and married are in the very-severe range. Additionally, the majority of both single and married participant fell in the normal range thus concluding that their marital status does not make a significant difference to their anxiety.

This study also focused on investigating the correlation between anxiety and carbohydrate-based food, where the majority of both carbohydrate consumers $(34 \%)$ and non-carbohydrate consumers (35\%) fell in the normal range of anxiety coming to the end result that carbohydrates do not affect an individual's level of anxiety.

This study further finds that amongst the participants that reported they are smokers, the majority (43\%) of them suffered from very severe anxiety whereas the majority of the non-smoking participants (34\%) fell in the normal range of anxiety indicating that smoking contributes to the severity of anxiety. These results are similar to another study that concluded that ingredients present in the cigarette smoke, like nicotine and also other toxic chemicals, exert influences on several pathways. These effects partly brace the biological mechanisms through which smoking tends to contribute to the advancement of anxiety $[19,20]$. When it comes to occupational status linked to anxiety level, this study shows that the students and employed participants mostly predominate in the normal anxiety range in comparison to the greater number of unemployed participants who ranged in severe to very severe anxiety implying that an individual's unemployment may be a major factor causing their anxiety.

\section{CONCLUSION}

Different lifestyle factors affect the levels of anxiety in various ways. Understanding these relations helps in avoiding the trigger factors of anxiety. This research clearly identified that a significant percentage of people who have any sort of psychological stress and do not exercise regularly are at a higher risk of developing or deteriorating anxiety. Gender, age, occupation, marital status, eating habits, sleep patterns, and smoking habits do not significantly play a huge role in the occurrence of anxiety. It is still advised to have an active and healthy lifestyle in order to prevent the incidence of psychological disorders like anxiety.

\section{AUTHORS CONTRIBUTIONS}

Dr Muaed Jamal Alomar: is the main researcher
Amal Mohamed Abdi: participated in the development of the methodology and data collection forms

Sumaiya Zaman: participated in the data collection and patients interviews

Hanadi Mohamed Abdi: participated in the data collection and patients interviews

Ma. Francheska Quicho: participated in the analysis and some of the literature review

\section{CONFLICT OF INTERESTS}

Declared none

\section{REFERENCES}

1. Walker R, Whittlesea C. Clinical pharmacy and therapeutics. Fifth edition; 2012. p. 454-64.

2. Goddard AW, Ball SG. Current perspectives of the roles of the central norepinephrine system in anxiety and depression. Depress Anxiety 2010;27:339-50.

3. Heim C, Nemerof CB. The impact of early adverse experiences on brain systems involved in the pathophysiology of anxiety and affective disorders. Biol Psychiatry 1999;46:1509-22.

4. Kroenke K, Spitzer RL, Williams JBW, Monahan PO, Lowe B. Anxiety disorders in primary care: prevalence, impairment, comorbidity, and detection. Ann Intern Med 2007;146:317-25.

5. Baxter AJ, Scott KM, Vos T, Whiteford HA. Global prevalence of anxiety disorders: a systematic review and meta-regression. Psychol Med 2012;43:897-910.

6. Lepine, Pierre J. The epidemiology of anxiety disorders: prevalence and societal costs. J Clin Psychiatry 2016;63:4-8.

7. Birkas B, Lang L, Martin L, Kallai J. Disturbing concerns for dark personalities: anxiety sensitivity and the dark triad. Int J Appl Pharm 2016;5:1-5.

8. Essau CA, Conradt J, Petermann F, Phil. Frequency, comorbidity, and psychosocial impairment of anxiety disorders in german adolescents. J Anxiety Disord 2000;12:263-79.

9. Pine DS, Fox NA. Childhood antecedents and risk for the adult mental disorder. Annu Rev Psychol 2015;66:459-85.

10. Mendlowicz MV, Stein MB. Quality of life in individuals with anxiety disorders. Am J Psychiatry 2000;157:669-82.

11. Stein MB, Kean YM. Disability and quality of life in social phobia: epidemiologic findings. Am J Psychiatry 2000;157:1606-13.

12. Ameringen MV, Mancini C, Farvolden P. The impact of anxiety disorders on educational achievement. J Anxiety Disord 2013;17:561-71.

13. Dixon T. Evolving self-confidence. How to Become Free from Anxiety Disorders and Depression; 2008.

14. Gupta V, Bansal P, Kumar P, Shri R. Anxiolytic effect of citrus paradisi var. marsh seedless using different models. Int Neuropsychiatr Dis J 2015;4:108-13.

15. Maes M, Song C, Lin A, Jongh RD, Gastel AV, Kenis G, et al. The effects of psychological stress on humans: Increased production of pro-inflammatory cytokines and Th1-like response in stress-induced anxiety. Cytokine 1998;10:313-8.

16. Mclean CP, Asnaani A, Ltiz BT, Hofmann SG. Gender differences in anxiety disorders: prevalence, the course of illness, comorbidity and burden of illness. J Psychiatr Res 2011;45:1027-35.

17. Babson KA, Trainor CD, Feldner MT, Blumenthal H. A test of the effects of acute sleep deprivation on general and specific selfreported anxiety and depressive symptoms: an experimental extension. J Behav Ther Exp Psychiatry 2010;41:297-303.

18. Jorm AF. Dose old age reduce the risk of anxiety and depression? A review of epidemiological studies across the adult lifespan. Psychol Med 2000;30:11-22.

19. Moylan S, Jacka FN, Pasco JA, Brek M. How cigarette smoking may increase the risk of anxiety symptoms and anxiety disorders: a critical review of biological pathways. Brain Behav 2013;3:302-26.

20. Sharma K, Harikumar SL. Recent advancement in drug delivery system for the brain: an overview. Int J Pharm Pharm Sci 2017;6:292-305. 